\title{
Upregulation of AUF1 is involved in the proliferation of esophageal squamous cell carcinoma through GCH1
}

\author{
YI GAO $^{1 *}$, WENJIE WANG ${ }^{2,3 *}$, JINMING CAO $^{2,3^{*}}$, FANGJUN WANG $^{1}$, YANGYANG GENG $^{2,3}$, \\ JIANPING CAO ${ }^{2,3}$, XIAOHUI XU ${ }^{4}$, JUNDONG ZHOU $^{5}$, PENGFEI LIU ${ }^{1}$ and SHUYU ZHANG ${ }^{2,3}$
}

\author{
${ }^{1}$ Department of Gastroenterology, The Jiangyin Clinical College of Xuzhou Medical University, Jiangyin, Jiangsu 214400; \\ ${ }^{2}$ School of Radiation Medicine and Protection and Jiangsu Provincial Key Laboratory of Radiation Medicine and Protection, \\ Medical College of Soochow University, Suzhou, Jiangsu 215123; ${ }^{3}$ Collaborative Innovation Center of Radiation \\ Medicine of Jiangsu Higher Education Institutions and School for Radiological and Interdisciplinary Sciences \\ (RAD-X), Soochow University, Suzhou, Jiangsu 215123; ${ }^{4}$ Department of General Surgery, The First People's \\ Hospital of Taicang, Soochow University, Taicang, Jiangsu 215400; ${ }^{5}$ The Core Laboratory of Suzhou Cancer \\ Center and Department of Radiotherapy of Suzhou Municipal Hospital, Suzhou, Jiangsu 215001, P.R. China
}

Received July 16, 2016; Accepted September 23, 2016

DOI: $10.3892 /$ ijo.2016.3713

\begin{abstract}
Esophageal squamous cell carcinoma (ESCC) has one of the highest mortality rates worldwide. AU-rich element RNA-binding factor 1 (AUF1) is an established RNA-binding protein. AUF1 influences the process of development, apoptosis and tumorigenesis via interacting with adenylate-uridylate rich elements (AREs) bearing mRNAs. However, the clinical relevance of AUF1 and its biological function in ESCC progression have not been reported. In the present study, we first investigated the expression of AUF1 in the ESCC tissue samles and normal samples. We found a significantly higher expression of AUF1 in ESCC tissues than that in normal tissues and tumor adjacent tissues. The expression of AUF1 correlated with ESCC stage $(\mathrm{P}=0.011)$ and marginally correlated with lymph node metastasis $(\mathrm{P}=0.055)$ of ESCC patients. Silencing of AUF1 by an siRNA inhibited the proliferation and enhanced the apoptosis of ESCC cells. mRNA profiling by microarray analysis revealed that AUF1 knockdown affected 285 genes (fold change $\geq 2$ ) that function
\end{abstract}

Correspondence to: Professor Pengfei Liu, Department of Gastroenterology, The Jiangyin Clinical College of Xuzhou Medical University, No. 163 Shoushan Road, Jiangyin, Jiangsu 214400, P.R. China

E-mail: pengfeimd@163.com

Professor Shuyu Zhang, School of Radiation Medicine and Protection and Jiangsu Provincial Key Laboratory of Radiation Medicine and Protection, Medical College of Soochow University, No. 199 Ren'ai Road, Suzhou, Jiangsu 215123, P.R. China

E-mail: zhang.shuyu@hotmail.com

${ }^{*}$ Contribured equally

Key words: esophageal squamous cell carcinoma, AU-rich element RNA-binding factor 1, 3'UTR, GTP cyclohydrolase I in multiple pathways. GTP cyclohydrolase I (GCH1), the rate limiting enzyme for $\mathrm{BH} 4$ synthesis, was found to be downregulated. One of the AU-rich elements in the 3'UTR of GCH1 was found to be responsive to AUF1 expression by luciferase assay. Knockdown of GCH1 suppressed cell proliferation and colony formation of ESCC cells. The expression of AUF1 significantly correlated with that of GCH1 in ESCC tissues. Taken together, we demonstrated the overexpression of AUF1 in esophageal carcinoma and identified GCH1 as AUF1's effector for the proliferation of ESCC cells.

\section{Introduction}

Esophageal cancer is the sixth leading cause of cancer death in male and the ninth in female, with an estimated over 400,000 new cases and deaths each year worldwide (1). The two main histological types are esophageal squamous cell carcinoma (ESCC) and esophageal adenocarcinoma. ESCC is the most common histological subtype in China with $95 \%$ of all esophageal cancer cases. With new methods of diagnosis and treatment, esophageal cancer still has a poor 5-year survival rate (2). Most of their mortality is not due to their primary tumors, but to their invasiveness and metastasis (3). A better understanding of molecular pathogenesis of ESCC is urgent for the diagnosis and treatment of the disease.

Regulation of gene expression is a major control point in organisms to adapt to the environment. The regulation of mRNA stability and translation are the basis of eukaryotes in response to intrinsic or extrinsic stimuli. Many mRNAs encode proto-oncogenes and cytokines, the aberrant mRNA stability is associated with inflammation and carcinogenesis $(4,5)$. It is recognized that approximately $16 \%$ of all human protein coding genes are encoded by mRNAs that contain an adenylate-uridylate (AU)-rich element (ARE) motif in their 3'UTR (6-9). AU-rich element RNA-binding factor 1 (AUF1), also called the heterogeneous nuclear ribonucleoprotein $\mathrm{D}$ (hnRNPD), is an established RNA-binding protein. The 
human AUF1 locus maps to 4q21, and it comprises a family of proteins composed of four isoforms with different molecular masses: p37, p40, p42 and p45 (10). AUF1 isoforms express preferentially in different tissues, which have their distinct biological properties (10). AUF1 influences the process of development, apoptosis and tumorigenesis via interacting with ARE-bearing mRNAs (11).

Altered AUF1 expression level may be a promoter to the development and progression of multiple types of cancers. It was reported that overexpression of AUF1 remarkably affected the stability of ARE containing mRNAs. Kumar et al (12) found that the expression level of nuclear AUF1 was significantly higher in oral squamous cell carcinoma than that in normal cells, and downregulated AUF1 with RNAi resulted in decreased proliferation rates. Overexpression of AUF1 isoform p37 developed sarcomas with deregulated c-Myc, c-Fos and c-Jun and cyclin D1 expression in tumor tissues (13). AUF1 has been shown to facilitate the decay of multiple target mRNAs, but occasionally to modulate the stability and translation of several target mRNAs (14). Several studies have shown that AUF1 plays an anti-tumorigenic role in regulation of the cell cycle and apoptosis. For example, increasing the AUF1 by UVC may promote apoptosis by reducing ARE-containing Bcl-2 mRNA (15). Treatment with postaglandin A2 (PGA2), an anticancer agent, induces expression of AUF1 isoform p45 and leads to the destabilization of cyclin D1 mRNA in non-small cell lung cancer cells (16). The above evidence suggests that AUF1 may be a promoter or inhibitor in the processes of carcinogenesis or proliferation, which vary between different types of cancer cells.

The clinical relevance of AUF1 and its biological function in ESCC progression have not been reported. In the present study, we investigated the expression of AUF1 in the ESCC tissue samles and normal samples. We further illustrated the role and downstream effector of AUF1 in driving cell proliferation of ESCC cells.

\section{Materials and methods}

Tissue samples. The ESCC samples were collected from 104 patients who had not received chemotherapy or radiotherapy prior to surgery. For these patients, if preoperative ultrasonography or computed tomography (CT) did not show any enlarged cervical lymph nodes (minor axis $<0.5 \mathrm{~cm}$ ), patients underwent a subtotal esophagectomy with two-field lymphadenectomy through a right thoracotomy, followed by a laparotomy. If enlarged cervical lymph nodes were detected by preoperative ultrasonography or CT, patients underwent radical oesophagectomy with cervico-thoraco-abdominal three-field lymphadenectomy through a right thoracotomy, followed by a laparotomy and a cervical incision. The resection extent includes nodes along the cervical part of the esophagus and deep cervix. Tumor adjacent tissues were obtained $3 \mathrm{~cm}$ away from the tumor. Fifteen normal esophageal tissue samples were obtained from surgical resections of trauma patients. These normal esophageal tissue samples were obtained from upper endoscopy of trauma patients, which were performed by the same operator. These patients had a fish bone or a poultry bone stuck. These tissues were obtained postoperatively between 2010 and 2013 from the Gastrointestinal Center,
Jiangyin People's Hospital, the Jiangyin Clinical College of Xuzhou Medical University (Jiangyin, China) as previously reported (17). All patients provided signed and informed consents for their tissue samples to be used for scientific research. The ethics approval for the study was obtained from the Jiangyin People's Hospital, the Jiangyin Clinical College of Xuzhou Medical University. All diagnoses were based on pathological and/or cytological evidence. The histological features of the specimens were evaluated by a senior pathologist according to the classification criteria from the World Health Organization (18). Tissue samples were obtained prior to chemotherapy and radiation therapy and were immediately fixed in $10 \%$ neutral buffered formalin prior to immunohistochemistry analysis.

Immunohistochemistry staining (IHC). The tissue samples were fixed in $10 \%$ neutral buffered formalin and embedded in paraffin. Three-micrometer thick paraffin sections were deparaffinized and heat-treated with citrate buffer ( $\mathrm{pH}$ 6.0) for $7 \mathrm{~min}$ as an epitope retrieval protocol. Endogenous peroxidase was blocked with $3 \%$ hydrogen peroxide for $15 \mathrm{~min}$ at room temperature and tissue non-specific binding sites were blocked with skim milk powder at $4 \%$ applied for $30 \mathrm{~min}$. Sections were then incubated with the AUF1 and GCH1 antibodies (Santa Cruz Biotechnology, Santa Cruz, CA, USA) for $1 \mathrm{~h}$ and mixed with skim milk powder at $2 \%$ again to reduce the non-specific staining. Biotinylated secondary antibody was then added for 30 min. IHC staining was visualized with substrate solution containing diaminobenzidine (DAB) and hydrogen peroxide. The counter-staining was performed with haematoxylin. All steps were performed at room temperature. Negative controls consisted of tissue sections undergoing similar staining procedures in the absence of the primary antibody. The criteria for scoring the stained sections were as follows: negative $(0),<10 \%$ of the whole tissue section stained positive; weakly positive (+ 1), $10-25 \%$ of the whole tissue section stained positive; moderately positive $(+2), 25-75 \%$ of the whole tissue section stained positive; and strongly positive $(+3), 75 \%$ of the tissue section stained positive. Tissues with score 0 or 1 were considered as AUF1-negative, while with score 2 or 3 were considered as AUF1-positive.

Cell culture and transfection. The human esophageal cancer cell line Eca-109 was maintained in Dulbecco's modified Eagle's medium (DMEM) supplemented with $10 \%$ fetal bovine serum (FBS) and antibiotics (Gibco, Grand Island, NY, USA). Cells were grown in a $37^{\circ} \mathrm{C}$ incubator with $5 \% \mathrm{CO}_{2}$.

The siRNA targeting AUF1 (siRNA-AUF1) and siRNA control (siRNA-NC) used in the present study were obtained from Santa Cruz Biotechnology. The cells were grown in a 6-well culture plate to 70-80\% confluence and then transfected with either siRNA-NC, siRNA-AUF1 or siRNA-GCH1 (Santa Cruz Biotechnology) using Lipofectamine 2000 (Invitrogen, Carlsbad, CA, USA) according to the manufacturer's instructions. After $48 \mathrm{~h}$, the cells were collected for western blot analysis.

Cell viability assay. Cell viability was evaluated using the 3-(4,5-dimethylthiazol-2-yl)-2,5-diphenyl-2H-tetrazolium bromide (MTT) assay. Cells were seeded in 96-well plates. 
Table I. Primer sequences for real-time PCR analysis.

\begin{tabular}{lll}
\hline Gene & \multicolumn{1}{c}{ Forward primer } & \multicolumn{1}{c}{ Reverse primer } \\
\hline GAPDH & 5'-AAGGTGAAGGTCGGAGTC-3' & 5'-GAAGATGGTGATGGGATTTC-3' \\
Nalcn & 5'-CCATGAAATCTGTCGTGTGC-3' & 5'-TGCTCACTGGACAATCAAGG-3' \\
BEP2 & 5' 2 & 5'-TTGCTCCATCTGTGCTTTTC-3' \\
$P r d m 16$ & 5'-TAATAAAGGGGAGCCCTTGG-3' & 5'-ACTGCCCGCAAACTATGACT-3' \\
& 5'-ACGCTTGGTTGAAGGTCACT-3' & 5'-TGGAAAAGTCGGAATGAACC-3'
\end{tabular}

The next day, the cells were transfected with plasmids or siRNAs according to the experimental design. The cells were then incubated with $20 \mu \mathrm{MTT}(5 \mathrm{mg} / \mathrm{ml})$ for $4 \mathrm{~h}$. After the medium was removed, $100 \mu 1$ DMSO was added and the optical density (OD) at $490 \mathrm{~nm}$ was measured using a microplate reader (Bio-Rad Laboratories, Hercules, CA, USA). The viability index was calculated as experimental OD value/control $\mathrm{OD}$ value. Three independent experiments were performed in quadruplicate.

Focus formation. Cells transfected with the indicated vectors were plated at low density (1,000 cells/6-cm plate), incubated for 10 days and fixed and stained with crystal violet. Foci and colonies containing $>50$ cells were counted using a microscope.

Western blot analysis. After transfection, cells were harvested and lysed in RIPA lysis buffer (50 mM Tris-HCl, $\mathrm{pH} \mathrm{7.4,}$ $150 \mathrm{mM} \mathrm{NaCl}, 1 \%$ Triton $\mathrm{X}-100,1 \%$ sodium deoxycholate, $0.1 \%$ SDS) containing protease inhibitor cocktail (Sigma-Aldrich, St. Louis, MO, USA) for $30 \mathrm{~min}$ at $4^{\circ} \mathrm{C}$. Protein $(40 \mu \mathrm{g})$ from each lysate was fractionated by $10 \%$ SDS-PAGE and transferred to polyvinylidene difluoride membranes (Millipore, Bedford, MA, USA). After blocking with 5\% non-fat milk in PBS-Tween-20 for $1 \mathrm{~h}$ at room temperature, the membranes were blotted with AUF1 and GCH1 primary antibodies (Abcam, Cambridge, UK). GAPDH (Beyotime Institute of Biotechnology, Nantong, China) was used as a loading control. After washing four times with TBST, the membranes were incubated with horseradish peroxidase (HRP)-conjugated secondary antibody for $2 \mathrm{~h}$. The proteins were visualized using the enhanced chemiluminescence (ECL; Beyotime Institute of Biotechnology).

Microarray analysis. Microarray-based mRNA expression profiling was performed using the Roche-NimbleGen (135K array) array (Roche NimbleGen, Madison, WI, USA). The microarrays contained approximately 45,033 assay probes corresponding to all of the annotated human mRNA sequences (NCBI HG18). Total RNA labeling and hybridization were performed using standard conditions according to the manufacturer's instructions. Genes with fold change of 2 or greater were subsequently subjected to pathway analysis using Ingenuity Pathway Analysis (Qiagen, Redwood City, CA, USA).

RNA extraction and real-time PCR. The total RNA from the esophageal tissue samples was extracted with TRIzol
(Invitrogen) and reverse transcribed to complementary DNA (cDNA) using an oligo(dT) $)_{12}$ primer and SuperScript II (Invitrogen). The SYBR-Green dye (Takara Bio, Osaka, Japan) was used for the amplification of cDNA. The mRNA levels of transcripts were measured using real-time quantitative PCR in triplicate on a Prism 7500 real-time PCR machine (Applied Biosystems, Foster City, CA, USA). Primers specific for the genes are listed in Table I. PCR was performed in a $20 \mu \mathrm{l}$ reaction mixture. The thermal cycling conditions included an initial denaturation step of $95^{\circ} \mathrm{C}$ for $20 \mathrm{sec}$, followed by 40 cycles at $95^{\circ} \mathrm{C}$ for $5 \mathrm{sec}$ and annealing at $60^{\circ} \mathrm{C}$ for $30 \mathrm{sec}$. The relative mRNA expression levels were normalized to $G A P D H$.

Luciferase assays. Two luciferase reporters containing the full-length 3'UTR fragment of the GCH1 3'UTR (+1 to +1063, relative to the stop coden) was constructed by Shanghai Biobuy Co., Ltd. (Shanghai, China). Then, constructs each with a deletion of the two potential AU-rich elements in the GCH1 3'UTR were constructed. For the luciferase assay, the reporter vector with the GCH1 3'UTR was co-transfected with pRL-TK (Promega, Madison, WI, USA) to correct the transfection efficiency. Luciferase activity was measured with the Dual-luciferase reporter assay system (Promega). Promoter activities were expressed as the ratio of firefly luciferase to Renilla luciferase activities.

Intracellular nitric oxide (NO) determination. Total NO concentration in cultured cells was detected by measuring the concentration of nitrate and nitrite by modified Griess reaction method. After lysis of cells, a total Nitric Oxide assay kit (Beyotime Institute of Biotechnology) was used to detect NO. The optical densities at $540 \mathrm{~nm}$ wavelength were recorded using a microplate reader (BioTek Instruments, Winooski, VT, USA) and the concentrations of NO were calculated according to the standard curve.

Statistical analysis. Data are expressed as the means \pm standard error of the mean (SEM) of at least three independent experiments. Standard error bars were included for all data points. The data were first analyzed with the KolmogorovSmirnov test for data distribution normality. The data were then analyzed using Student's t-test when only two groups were present or assessed by one-way analysis of variance (ANOVA) when more than two groups were compared. The P-values for t-tests were performed by 2-tailed t-tests. Correlation analysis of the mRNA expression data was performed using the Pearson test. Statistical analysis was 
Table II. Patient demographic features for the IHC analysis.

\begin{tabular}{lcccccc}
\hline & Normal & Grade I & Grade II & Grade III & Grade IV & All tumors \\
\hline Number & 15 & 15 & 52 & 33 & 4 & 104 \\
Mean age (years) & 58.73 & 62.88 & 59.21 & 56.50 & 66.00 & 58.90 \\
Age range (years) & $32-65$ & $55-69$ & $33-73$ & $32-75$ & $52-67$ & $32-76$ \\
Gender & & & & & & \\
Male & 10 & 9 & 37 & 23 & 4 & 73 \\
Female & 5 & 6 & 15 & 10 & 0 & 31 \\
\hline
\end{tabular}

A

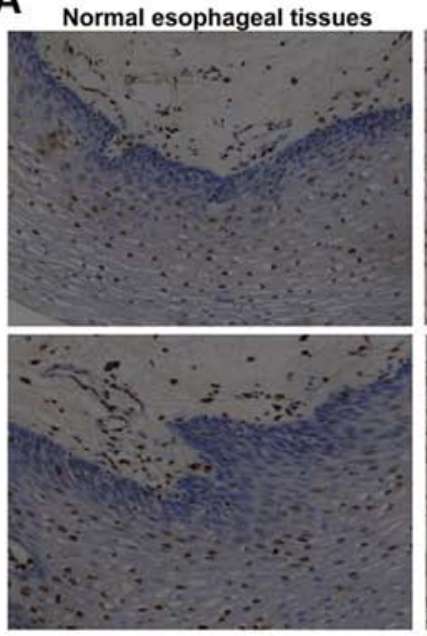

Tumor tissues

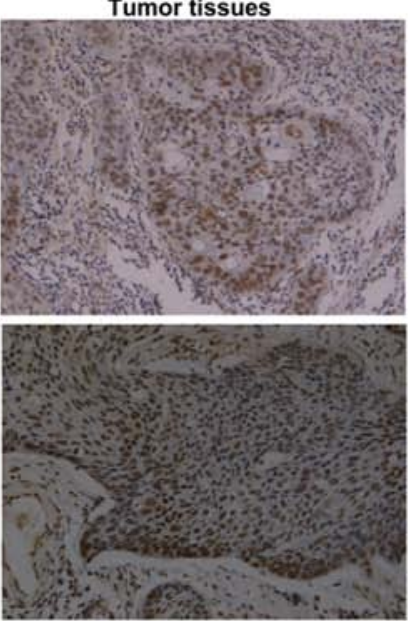

B

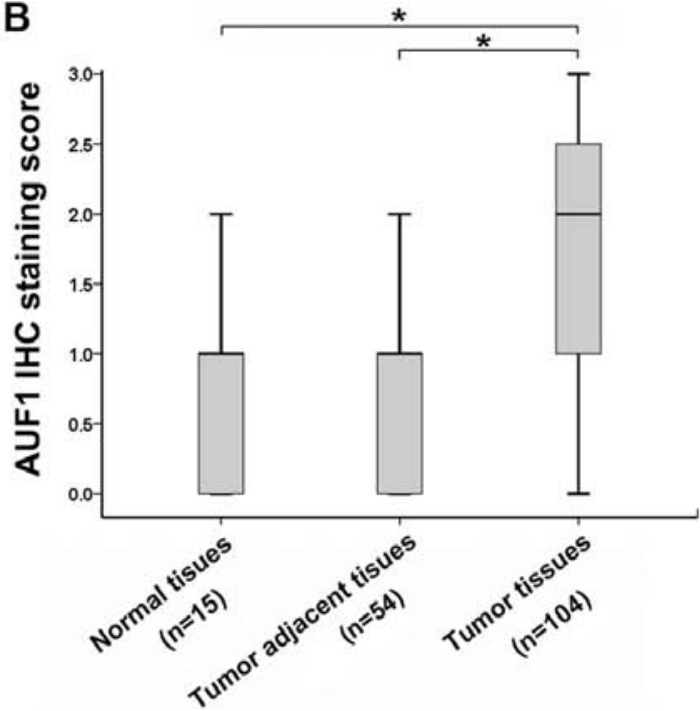

Figure 1. Upregulation of AUF1 in ESCC samples. (A) Representative IHC staining of AUF1 expression in normal esophageal tissue specimen and ESCC tissues (magnification, x100). (B) Box plot representing the range of AUF1 immunohistochemical (IHC) staining score in normal esophageal tissues ( $\mathrm{n}=15$ ), ESCC tissues $(n=104)$ and tumor adjacent tissues $(n=52) .{ }^{*} P<0.05$.

performed using SPSS software (Release 19.0; SPSS, Inc., Chicago, IL, USA). Data were considered significant at $\mathrm{P}<0.05$.

\section{Results}

Upregulation of AUF1 is correlated with clinicopathological feature in esophageal squamous cell carcinoma. To investigate the expression of AUF1 in human ESCC tissues, we performed immunohistochemistry analysis on tissue section of normal esophageal tissue specimens, ESCC and tumor adjacent tissues. Fifteen paraffin-embedded normal esophageal tissues and 104 ESCC tissues, 54 of which had corresponding adjacent tissues were collected. The demographic features of patients with ESCC for the immunohistochemistry staining are summarized in Table II. Results from IHC staining showed that a generally stronger staining of AUF1 was observed in the nucleus of ESCC tissues, whereas very weak staining of AUF1 was observed in the normal samples (Fig. 1A). When the samples were scored by a pathologist, as described in Materials and methods, AUF1 expression was similar between normal esophageal tissues and tumor adjacent tissues, while increased significantly among ESCC tissues, and between tumor tissues and tumor adjacent tissues $(\mathrm{P}<0.05$; Fig. 1B). All the above results indicate that AUF1 may be involved in the pathogenesis of ESCC.

Next, the relationship between clinicopathology of ESCC patients and AUF1 IHC staining score was analyzed. As shown in Table III, the results showed a non-significant correlation between AUF1 expression and age $(\mathrm{P}=0.610)$, gender $(\mathrm{P}=0.637)$ and lymphatic invasion $(\mathrm{P}=0.096)$ of ESCC patients. AUF1 expression correlated with ESCC stage $(\mathrm{P}=0.011)$ and marginally correlated with lymph node metastasis $(\mathrm{P}=0.055)$. These results suggest $\mathrm{AUF1}$ as a characteristic of this malignancy.

AUF1 expression affects the proliferation and apoptosis of ESCC cells. Because AUF1 expression was associated with ESCC progression, we sought to explore the role of AUF1 in esophageal cancer cell growth. Eca-109 cells were transfected with a siRNA control (siRNA-NC) or siRNA trageting AUF1 (siRNA-AUF1). As shown in Fig. 2A, the protein level of AUF1 was markedly reduced after transfection of siRNA-AUF1. Next, the effect of AUF1 on cell growth was measured with MTT and colony formation. The results showed that decreased AUF1 expression suppressed cell growth and focus formation (Fig. 2B and C). We next investigated whether reduced cell growth was associated with apoptosis in ESCC cells. As 
A

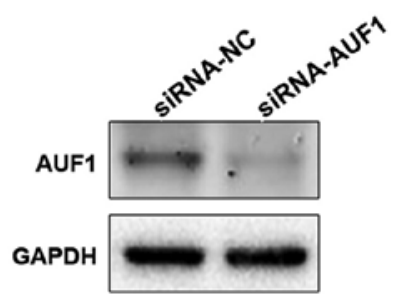

B

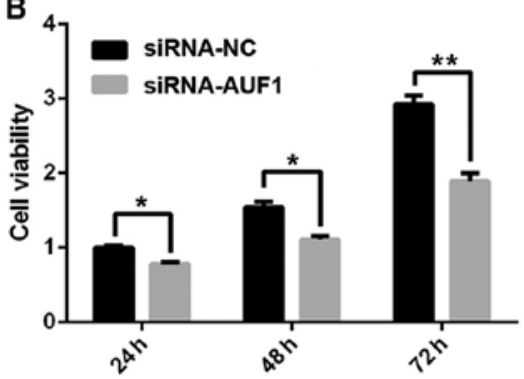

C

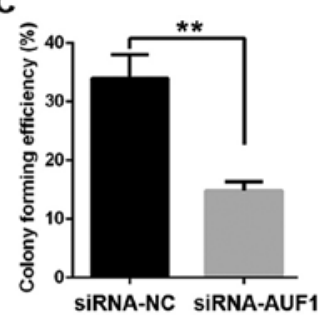

E

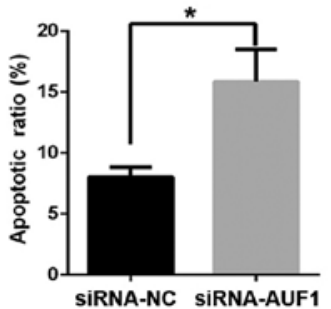

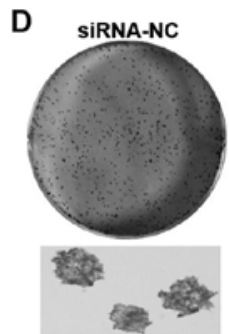
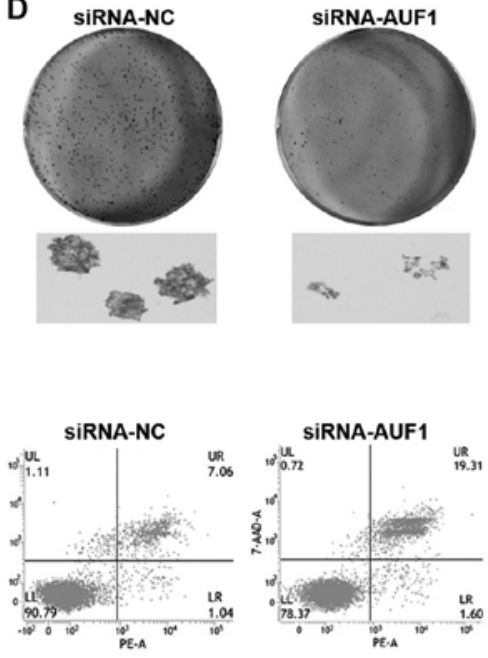

Figure 2. The effect of AUF1 on cell proliferation and focus formation. (A) Eca-109 cells were transfected with siRNA-AUF1 or siRNA-NC. The protein level of AUF1 was measured by western blot analysis. (B) Eca-109 cell lines were seeded in a 96 -well plate and incubated for 48 h. Cells were transfected with siRNA-control (siRNA-NC) or siRNA-AUF1. Cell proliferation was measured by MTT. (C and D) The effect of AUF1 on Eca-109 cell focus formation. Eca-109 cells were transfected with siRNA-AUF1 or siRNA-NC. One thousand cells were seeded onto each plate. After 10 days, cells were stained with crystal violet. Colonies consisting of $>50$ cells were counted. The upper panel shows the representative focus, and the lower panel shows the representative clones. (E) Cell apoptosis rate was measured using Annexin V/7-AAD double staining. Data are presented as means \pm SEM and were normalized to the control cells. ${ }^{*} \mathrm{P}<0.05$ and ${ }^{* *} \mathrm{P}<0.01$, compared with the control group.

Table III. Relationship between clinicopathological parameters and the expression of AUF1 in esophageal squamous cell carcinoma (ESCC) $(n=104)$.

\begin{tabular}{|c|c|c|c|c|}
\hline \multirow{2}{*}{$\begin{array}{l}\text { Clinicopathological } \\
\text { parameters }\end{array}$} & \multirow[b]{2}{*}{ Case } & \multicolumn{2}{|c|}{ AUF1 expression } & \multirow[b]{2}{*}{ P-value } \\
\hline & & Negative & Positive & \\
\hline Age (years) & & & & 0.610 \\
\hline$<64$ & 61 & 22 & 39 & \\
\hline$\geq 64$ & 43 & 13 & 30 & \\
\hline Gender & & & & 0.637 \\
\hline Male & 73 & 25 & 48 & \\
\hline Female & 31 & 10 & 21 & \\
\hline Stage & & & & 0.011 \\
\hline 1 & 15 & 9 & 6 & \\
\hline 2 & 52 & 18 & 34 & \\
\hline 3 & 33 & 7 & 26 & \\
\hline 4 & 4 & 1 & 3 & \\
\hline $\mathrm{pN}$ & & & & 0.055 \\
\hline$(+)$ & 41 & 9 & 32 & \\
\hline$(-)$ & 63 & 26 & 37 & \\
\hline Lymphatic invasion & & & & 0.096 \\
\hline$(+)$ & 49 & 12 & 37 & \\
\hline$(-)$ & 55 & 23 & 32 & \\
\hline
\end{tabular}

$\mathrm{pN}$, lymph node metastasis.

shown in Fig. 2E, silencing of AUF1 significantly increased apoptosis of Eca-109 cells. These results demonstrated that
AUF1 modulates the proliferation and apoptotic cell death of ESCC cells.

mRNA microarray screening of AUFl-affected genes. Although many AUF1-regulated genes have been identified, the profile of AUF1-affected genes in ESCC cells remains elusive. To further analyze the underlying mechanisms responsible for AUF1-mediated effects, we screened gene expression between AUF1 silencing (siRNA-AUF1) and the control cells (siRNA-NC) by microarray. Twenty-four hours after the transfection, a total of 285 genes (138 upregulated and 147 downregulated genes) were identified with an expression differential of 2-fold or greater between the two groups ( $\mathrm{n}=2$; Fig. 3A and Table IV). The differentially expressed genes included Nalcn, MMP23B, E2F1, BEX2 and GCH1. We then validated the results from microarray analysis by RT-PCR. As shown in Fig. 3B, compared with control cells, several genes dysregulated in microarray data, such as Nalcn, UGP2, BEX2 and Prdm16 showed consistent changes by real-time PCR analysis. As expected, AUF1 appeared to have modulated the Eca-109 cells via complex mechanisms. Pathway analysis revealed that AUF1 affected multiple pathways, including MAPK signaling pathway, transcriptional regulation and adipocytokine signaling pathway (Fig. 3C). These results suggested that AUF1 might be a wide-spectrum regulator of gene expression in esophageal cancer cells.

AUF1 regulated GCH1 expression through 3'UTR. Among AUF1 affected genes, we focused on GCH1, which catalyzes the formation of 5,6,7,8-tetrahydrobiopterin (BH4), an essential co-factor for all nitric oxide synthases $(19,20)$. GCH1 has been reported to contribute to cancer progression (21). Sequence analysis of the GCH1 3'UTR identified two potential AU-rich 
A

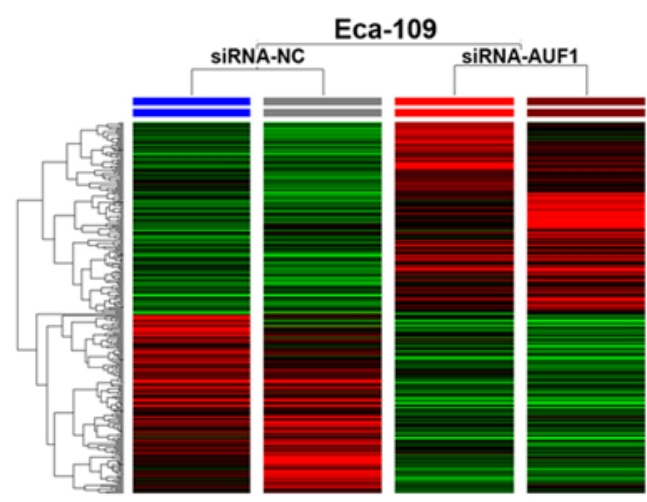

B

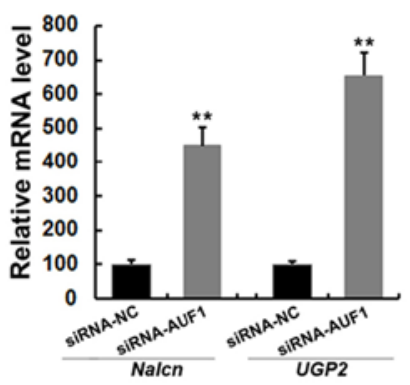

C
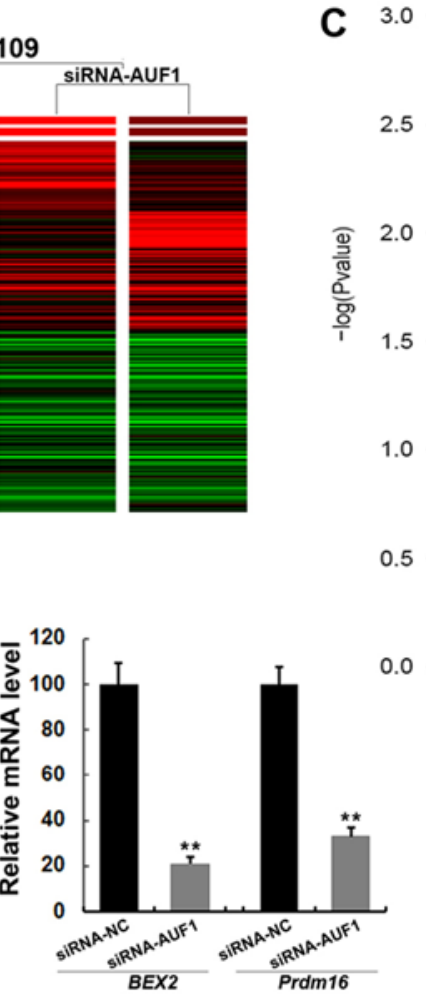

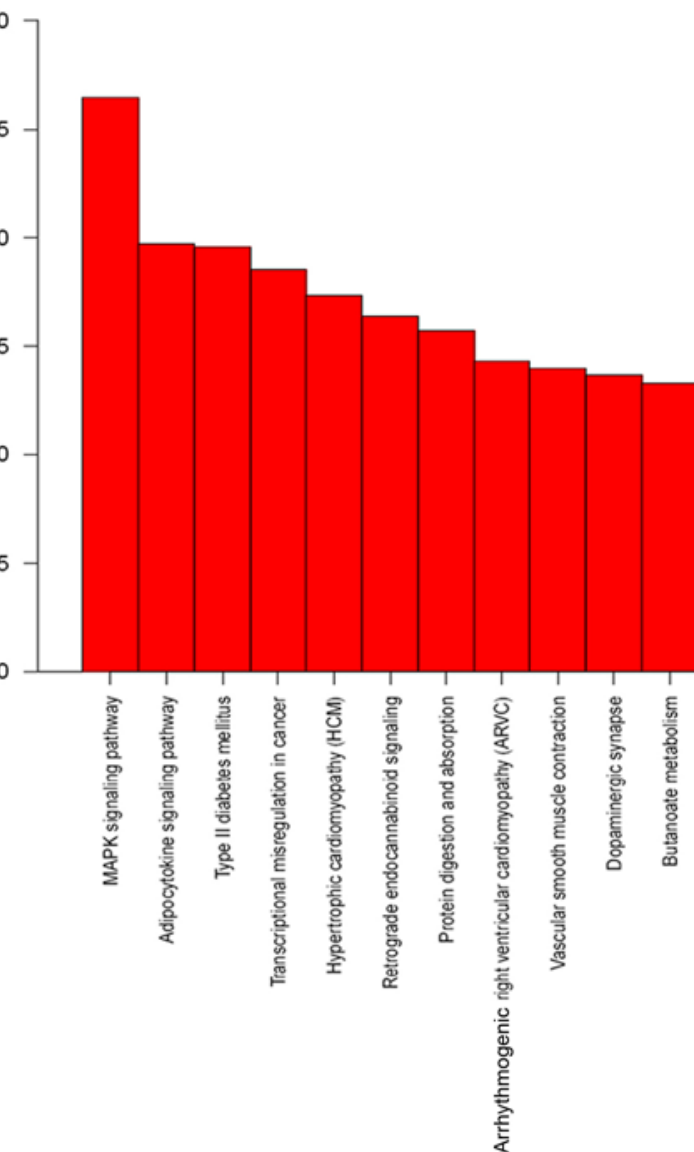

Figure 3. Predicted significant pathways affected by AUF1 overexpression. Cells were transfected with siRNA-AUF1 or siRNA-NC. RNA samples were collected and subjected to mRNA micorarray analysis. (A) Heatmap of gene expression between siRNA-NC- and siRNA-AUF1-transfected Eca-109 cells. (B) Real-time PCR analysis of Nalcn, UGP2, BEX2 and Prdm 16 in the control and AUF1 silenced Eca-109 cells. (C) Predicted significant pathways for dysregulated genes.

A

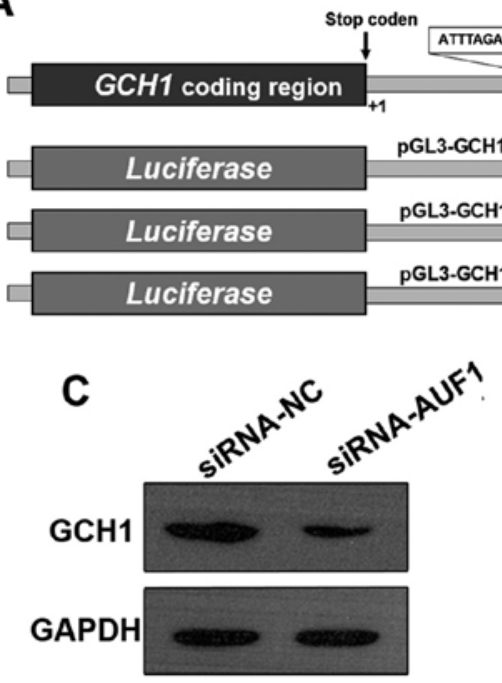

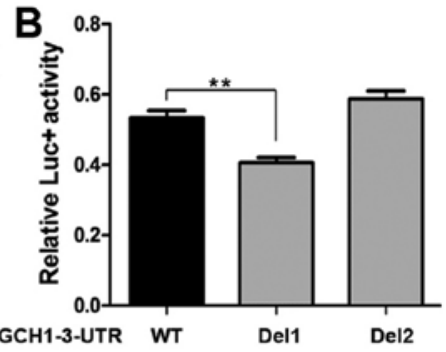

D

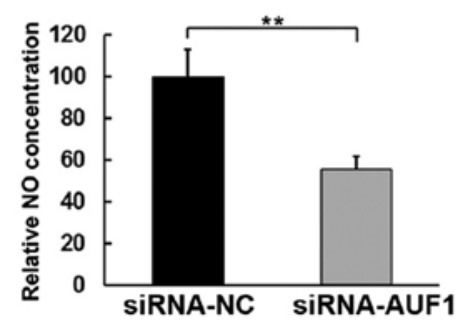

Figure 4. AUF1 positively regulates GCH1 in esophageal cancer cells. (A) The diagram indicates the relative positions of potential AU-rich elements (ARE) in the GCH1 3'UTR. Wild-type GCH1 3'UTR or deletion of each ARE in GCH1 3'UTR were cloned downstream of the firefly luciferase reporter gene. (B) Eca-109 cells were transfected with equivalent indicated reporters. After $24 \mathrm{~h}$ of incubation, luciferase activity was measured using the Dual-luciferase reporter assay system (Promega). (C) Eca-109 cells were transfected with siRNA-NC or siRNA-AUF1. Western blot analysis of GCH1 expression after silencing of AUF1. (D) The NO concentration was measured using the Griess assay. ${ }^{* *} \mathrm{P}<0.01$ compared with the control group.

elements (Fig. 4A), which were likely to be regulated by AUF1. We therefore investigated whether AUF1 modulated the expression of GCH1 and cancer progression through GCH1. The effect of AUF1 on the GCH1 3'UTR was also measured 
Table IV. Micorarray analysis of gene expression changes between siRNA-NC- and siRNA-AUF1-transfected cells.

\begin{tabular}{|c|c|c|c|}
\hline Gene name & Fold change upregulated & Chromosome & Description \\
\hline NALCN & 25.949 & $\operatorname{chr} 13$ & Sodium leak channel, non selective \\
\hline UGP2 & 20.386 & $\operatorname{chr} 2$ & UDP-glucose pyrophosphorylase 2 \\
\hline SCAF1 & 17.645 & $\operatorname{chr} 19$ & SR-related CTD-associated factor 1 \\
\hline KRTAP10-10 & 17.180 & $\operatorname{chr} 21$ & Keratin associated protein $10-10$ \\
\hline MMP23B & 16.273 & chr1 & Matrix metallopeptidase 23B \\
\hline MAP7D2 & 15.894 & $\operatorname{chrX}$ & MAP7 domain containing 2 \\
\hline TMEM129 & 15.310 & $\operatorname{chr} 4$ & Transmembrane protein 129 , E3 ubiquitin protein ligase \\
\hline DACT1 & 15.130 & $\operatorname{chr} 14$ & Dishevelled-binding antagonist of $\beta$-catenin 1 \\
\hline ARHGAP30 & 13.292 & chr1 & Rho GTPase activating protein 30 \\
\hline $\mathrm{E} 2 \mathrm{~F} 1$ & 13.152 & $\operatorname{chr} 20$ & E2F transcription factor 1 \\
\hline PCNXL2 & 12.669 & chr1 & Pecanex-like 2 (Drosophila) \\
\hline NID1 & 12.587 & chr1 & Nidogen 1 \\
\hline PAK6 & 11.838 & $\operatorname{chr} 15$ & P21 protein $(\mathrm{Cdc} 42 / \mathrm{Rac})$-activated kinase 6 \\
\hline AMBRA1 & 11.740 & $\operatorname{chr} 11$ & Autophagy/Beclin-1 regulator 1 \\
\hline TAF11 & 11.715 & chr6 & $\begin{array}{l}\text { TAF11 RNA polymerase II, TATA Box binding protein } \\
\text { (TBP)-associated factor, } 28 \mathrm{kDa}\end{array}$ \\
\hline NCDN & 11.146 & chr1 & Neurochondrin \\
\hline C14ORF101 & 11.047 & $\operatorname{chr} 14$ & Transmembrane protein 260 \\
\hline WDR20 & 10.609 & $\operatorname{chr} 14$ & WD repeat domain 20 \\
\hline CCDC74A & 10.475 & $\operatorname{chr} 2$ & Coiled-coil domain containing 74A \\
\hline TRO & 10.257 & $\operatorname{chrX}$ & Trophinin \\
\hline
\end{tabular}

\begin{tabular}{|c|c|c|c|}
\hline Gene name & Fold change downregulated & Chromosome & Description \\
\hline $\mathrm{BEX} 2$ & 23.010 & $\operatorname{chrX}$ & Brain expressed X-linked 2 \\
\hline PRDM16 & 19.479 & $\operatorname{chr} 1$ & PR domain containing 16 \\
\hline ARX & 18.122 & $\operatorname{chrX}$ & Aristaless related homeobox \\
\hline CPZ & 17.834 & chr4 & Carboxypeptidase Z \\
\hline GRM4 & 17.232 & chr6 & Glutamate receptor, metabotropic 4 \\
\hline PHF20L1 & 16.919 & chr8 & PHD finger protein 20 -like 1 \\
\hline ANO9 & 13.588 & chr11 & Anoctamin 9 \\
\hline SLC24A4 & 13.212 & $\operatorname{chr} 14$ & Solute carrier family 24 \\
\hline BAZ1B & 12.677 & $\operatorname{chr} 7$ & Bromodomain adjacent to zinc finger domain, 1B \\
\hline C1ORF147 & 11.959 & $\operatorname{chr} 1$ & Chromosome 1 open reading frame 147 \\
\hline SIRT2 & 10.788 & chr19 & Sirtuin 2 \\
\hline RCBTB1 & 10.731 & $\operatorname{chr} 13$ & $\begin{array}{l}\text { Regulator of chromosome condensation ( } \mathrm{RCC} 1) \text { and } \\
\text { BTB (POZ) domain containing protein } 1\end{array}$ \\
\hline ISM1 & 10.317 & $\operatorname{chr} 20$ & Isthmin 1 , angiogenesis inhibitor \\
\hline OAZ2 & 10.129 & $\operatorname{chr} 15$ & Ornithine decarboxylase antizyme 2 \\
\hline SNCAIP & 10.088 & $\operatorname{chr} 5$ & Synuclein, $\alpha$ interacting protein \\
\hline CAMK1G & 10.031 & $\operatorname{chr} 1$ & Calcium/calmodulin-dependent protein kinase IG \\
\hline MYCT1 & 9.578 & chr6 & Myc target 1 \\
\hline SEC14L3 & 8.865 & $\operatorname{chr} 22$ & SEC14-like lipid binding 3 \\
\hline SGCB & 8.732 & $\operatorname{chr} 4$ & Sarcoglycan, $\beta$ \\
\hline GCH1 & 8.726 & $\operatorname{chr} 14$ & GTP cyclohydrolase 1 \\
\hline
\end{tabular}

using luciferase constructs containing the GCH1 3'UTR with or without AUF1 binding sites (Fig. 4A). The results revealed that deletion of the ARE1 (pGL3-GCH1 3UTR Del1) showed significantly decreased luciferase activity compared with that of wild-type GCH1 3'UTR in Eca-109 cells (Fig. 4B). However, deletion of the ARE2 (pGL3-GCH1 3UTR Del2) showed similar luciferase activity as compared with that of wild-type GCH1 3'UTR in Eca-109 cells (Fig. 4B). Western 
A

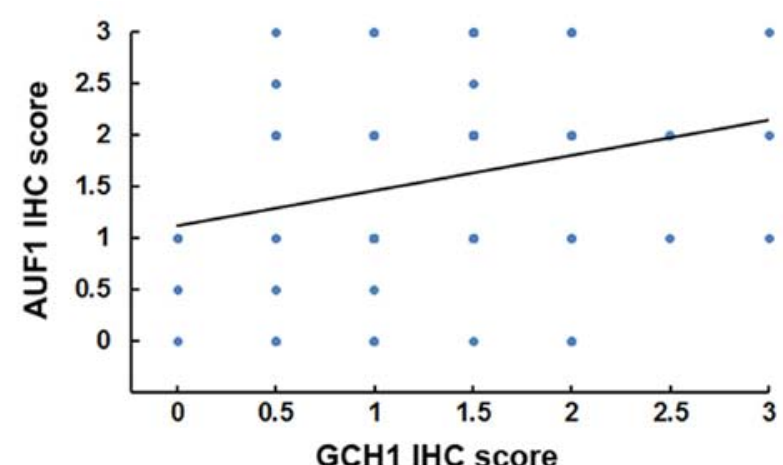

B

B GCH1 IHC score
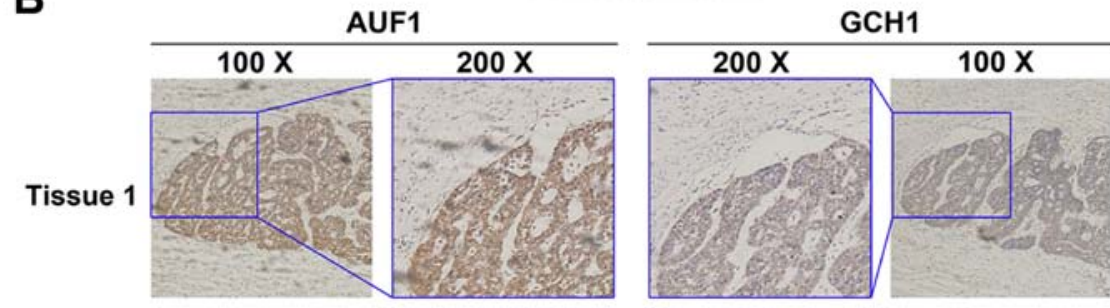

Tissue 2
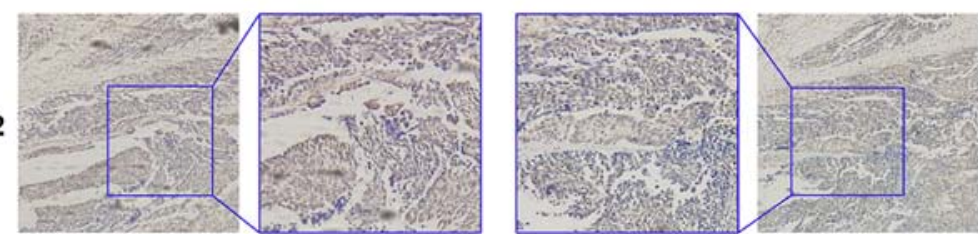

Figure 5. Correlation analyses between AUF1 and GCH1 expression in tissue samples. Immunohistochemistry (IHC) was used to detect the expression levels of AUF1 and GCH1 tissue samples. (A) Correlation between AUF1 and GCH1 in 104 ESCC tissue samples. AUF1 positively correlated with GCH1 in esophageal tissue samples. (B) Representative IHC staining of both AUF1 and GCH1 in the same tissue.
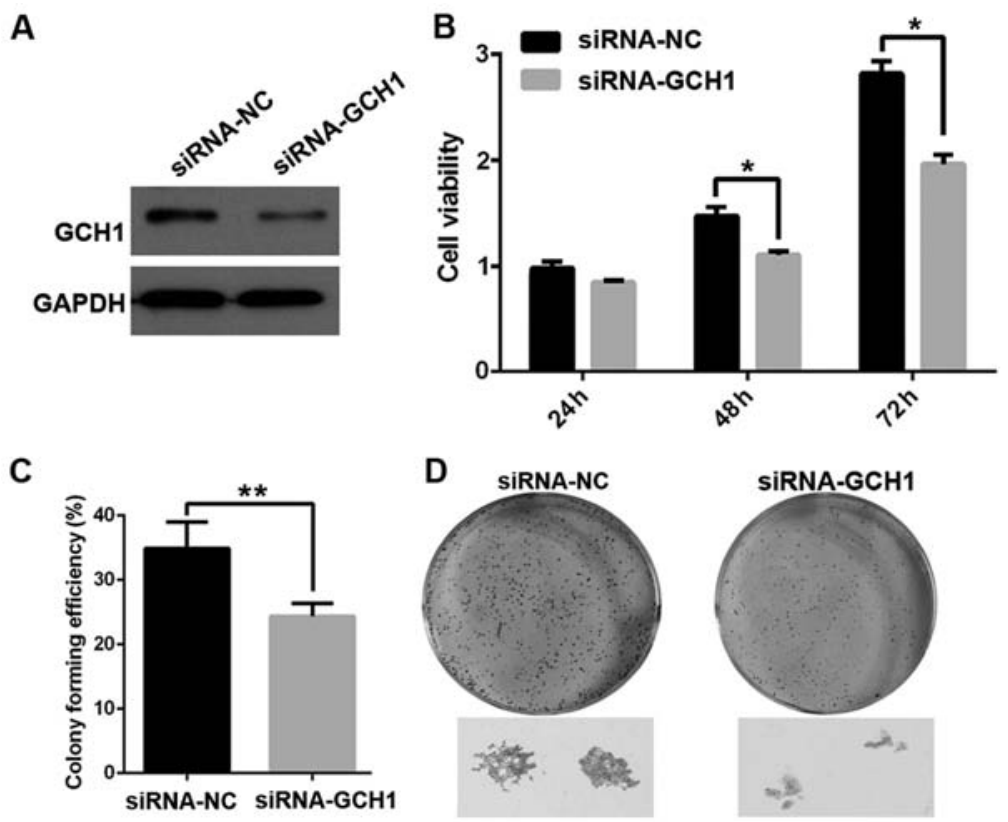

Figure 6. The effect of GCH1 on cell proliferation and focus formation of ESCC cells. Eca-109 cells were seeded in a 96-well plate and incubated for $48 \mathrm{~h}$. (A) Cells were transfected with siRNA-control (siRNA-NC) or siRNA-GCH1. (B) After the transfection, cell proliferation was measured by MTT. Data are presented as means \pm SEM and were normalized to the control cells, ${ }^{*} \mathrm{P}<0.05 ;{ }^{* *} \mathrm{P}<0.01$. (C and D) The effect of AUF1 on Eca-109 cell focus formation. Eca-109 cells were transfected with siRNA-control (siRNA-NC) or siRNA-GCH1. One thousand cells were seeded onto each plate. After 10 days, cells were stained with crystal violet. Colonies consisting of more than 50 cells were counted. The upper panel shows the representative focus, and the lower panel shows the representative clones.

blot analysis further confirmed that knockdown of AUF1 decreased the expression of GCH1 (Fig. 4C). Because GCH1 is an essential co-factor for all NOS, as expected, silencing of AUF1 significantly reduced cellular NO level. These results 
suggested that AUF1 regulated GCH1 through ARE1 region (Fig. 4D).

Expression of AUF1 correlates with GCH1 in ESCC tissues. The above mentioned results led us to investigate whether the expression level of AUF1 correlated with that of $\mathrm{GCH} 1$ in tissue samples. Immunohistochemistry was used to detect the expression levels of GCH1 protein in 102 ESCC tissue samples. Representative IHC staining of both AUF1 and GCH1 in the same tissue is shown in Fig. 5A. Tissue samples with strong AUF1 staining in the nucleus exhibited positive GCH1 staining, whereas weak AUF1 expression showed negative GCH1 staining. Statistical analysis revealed that the protein levels of the two genes correlated with each other (Pearson $r=0.251, P=0.019$; Fig. 5B), indicating that the expression of AUF1 correlates with the expression of $\mathrm{GCH} 1$ in human ESCC tissues.

Inhibition of GCH1 suppresses the proliferation and colony formation in ESCC cells. To explore the role of GCH1 in esophageal cancer cell growth, cells were first transfected with a siRNA-NC or siRNA-GCH1, and the resulting growth was measured with MTT and colony formation assay. The results showed that GCH1 silencing caused significantly decreased cell viability ( $75 \%$ of the control group). Moreover, knockdown of GCH1 attenuated the number and the size of colonies of Eca-109 cells. These results suggest that $\mathrm{GCH} 1$ promotes esophageal tumor growth in vitro. (Fig. 6).

\section{Discussion}

AUF1 plays a central role in various physiological and pathological processes. Excessive and dysregulated AUF1 expression has been implicated in diseases including cancers. Overexpression of AUF1 has been detected in numerous malignancies, including breast, skin, thyroid, liver, oral squamous cell carcinoma and sarcoma $(12,13,16,22-24)$. AUF1 has therefore been recognized to modulate different cancer-related events. However, there is considerable controversy in defining its role in cancer progression. Several studies have shown that AUF1 has both tumor promoting and tumoricidal effects depending on cancer types (12-16). AUF1 has been suggested to modulate different cancer-related events including apoptosis, tumorigenesis and development (10). However, the role of AUF1 in ESCC progression has not been reported. In the present study, we found that the expression of AUF1 was significantly higher in ESCC tissues than that in normal tissues and tumor adjacent tissues. In this study, we found that AUF1 levels are correlated with tumor stage, indicating that AUF1 may be a predicator of malignant degree, and related with prognosis of the malignancy. We also found that AUF1 silencing resulted in markedly decreased cell proliferation and focus formation and elevated cell apoptosis.

AUF1 has been implicated in regulating the expression of numerous mRNAs containing 3'UTR ARE, including $\mathrm{Bcl} 2$, cyclin D1 and SOD1 $(10,13,14)$. However, the regulatory profile of AUF1 may be cell-specific. To comprehensively understand the function of AUF1 in esophageal cancer, we performed mRNA microarray-based screening analysis in Eca-109 cells. Through this analysis, we found that overexpression of AUF1 affected multiple genes, such as Nalcn, UGP2, BEX2 and Prdm16. Among AUF1 affected genes, GCH1 encoded protein is the first and rate-limiting enzyme in tetrahydrobiopterin (BH4) biosynthesis, catalyzing the conversion of GTP into 7,8-dihydroneopterin triphosphate (25). BH4 is an essential element required for nitric oxide (NO) formation by all nitric oxide synthases (NOSs) (26). NO has been reported to modulate many cancer-related events, such as angiogenesis, apoptosis, cell cycle, invasion and metastasis (27), and correlated with the development of several cancers, including breast, cervical, lung, gastric and head and neck cancer (28-33). Therefore, GCH1 is likely to play important roles in cancer progression. Chen et al (34) reported that GCH1 promotes cancer angiogenesis in vitro and in vivo. Consistently, another group demonstrates that $\mathrm{GCH} 1$ inhibition reduces tumor growth by: i) direct killing of tumor cells; ii) inhibiting angiogenesis; and iii) enhancing the antitumoral immune response. However, little is known about the functional significance of GCH1 in ESCC cancer. Here, we report that GCH1 was regulated by AUF1 and involved in the proliferation and colony formation in ESCC cells. The function of GCH1 in other aspects and regulatory mechnisms merit further investigation.

In summary, we found that AUF1 expression was significantly increased in ESCC tumor tissues, and that its expression correlated with tumor stage in human ESCC tissue samples. Furthermore, knockdown of AUF1 resulted in markedly decreased proliferation and increased cell apoptosis. AUF1 silencing-affected genes were screened by microarray assay. In particular, we demonstrated that AUF1 regulated the expression of GCH1 through its 3'UTR. Silencing of GCH1 inhibited the growth of ESCC cells. In conclusion, AUF1/GCH1 axis may present a novel potential target in the treatment of ESCC.

\section{Acknowledgements}

The present study was supported by the Natural Science Foundation of Jiangsu Province (BK20161152), the National Natural Science Foundation of China (31670857, 81672975, 81522039 and 31400720), the Key Research and Development Program of China (2016YFC0904702) and the Key Scientific Research Program of Wuxi Municipal Health Bureau (Z201509).

\section{References}

1. Siegel R, Naishadham D and Jemal A: Cancer statistics, 2013. CA Cancer J Clin 63: 11-30, 2013.

2. Enzinger PC and Mayer RJ: Esophageal cancer. N Engl J Med 349: 2241-2252, 2003.

3. Ji X, Cai J, Chen Y and Chen LQ: Lymphatic spreading and lymphadenectomy for esophageal carcinoma. World $\mathbf{J}$ Gastrointest Surg 8: 90-94, 2016.

4. Wilusz CJ, Wormington M and Peltz SW: The cap-to-tail guide to mRNA turnover. Nat Rev Mol Cell Biol 2: 237-246, 2001.

5. Pont AR, Sadri N, Hsiao SJ, Smith S and Schneider RJ: mRNA decay factor AUF1 maintains normal aging, telomere maintenance, and suppression of senescence by activation of telomerase transcription. Mol Cell 47: 5-15, 2012.

6. Gruber AR, Fallmann J, Kratochvill F, Kovarik P and Hofacker IL: AREsite: A database for the comprehensive investigation of AU-rich elements. Nucleic Acids Res 39 (Database): D66-D69, 2011.

7. White EJ, Brewer G and Wilson GM: Post-transcriptional control of gene expression by AUF1: Mechanisms, physiological targets, and regulation. Biochim Biophys Acta 1829: 680-688, 2013. 
8. Gratacós FM and Brewer G: The role of AUF1 in regulated mRNA decay. Wiley Interdiscip Rev RNA 1: 457-473, 2010.

9. Barker A, Epis MR, Porter CJ, Hopkins BR, Wilce MC, Wilce JA, Giles KM and Leedman PJ: Sequence requirements for RNA binding by HuR and AUF1. J Biochem 151: 423-437, 2012.

10. Moore AE, Chenette DM, Larkin LC and Schneider RJ Physiological networks and disease functions of RNA-binding protein AUF1. Wiley Interdiscip Rev RNA 5: 549-564, 2014.

11. Abdelmohsen K, Tominaga-Yamanaka K, Srikantan S, Yoon JH, Kang MJ and Gorospe M: RNA-binding protein AUF1 represses Dicer expression. Nucleic Acids Res 40: 11531-11544, 2012.

12. Kumar M, Matta A, Masui O, Srivastava G, Kaur J, Thakar A, Shukla NK, RoyChoudhury A, Sharma M, Walfish PG, et al: Nuclear heterogeneous nuclear ribonucleoprotein D is associated with poor prognosis and interactome analysis reveals its novel binding partners in oral cancer. J Transl Med 13: 285, 2015.

13. Gouble A, Grazide S, Meggetto F, Mercier P, Delsol G and Morello D: A new player in oncogenesis: AUF1/hnRNPD overexpression leads to tumorigenesis in transgenic mice. Cancer Res 62: 1489-1495, 2002.

14. von Roretz C, Di Marco S, Mazroui R and Gallouzi IE: Turnover of AU-rich-containing mRNAs during stress: A matter of survival. Wiley Interdiscip Rev RNA 2: 336-347, 2011.

15. Lapucci A, Donnini M, Papucci L, Witort E, Tempestini A, Bevilacqua A, Nicolin A, Brewer G, Schiavone $\mathrm{N}$ and Capaccioli S: AUF1 is a $b c l-2 \mathrm{~A}+\mathrm{U}$-rich element-binding protein involved in $b c l-2$ mRNA destabilization during apoptosis. J Biol Chem 277: 16139-16146, 2002

16. Lin S, Wang W, Wilson GM, Yang X, Brewer G, Holbrook NJ and Gorospe M: Down-regulation of cyclin D1 expression by prostaglandin $A_{2}$ is mediated by enhanced cyclin D1 mRNA turnover. Mol Cell Biol 20: 7903-7913, 2000.

17. Luo J, Zhou X, Ge X, Liu P, Cao J, Lu X, Ling Y and Zhang S: Upregulation of Ying Yang 1 (YY1) suppresses esophageal squamous cell carcinoma development through heme oxygenase-1. Cancer Sci 104: 1544-1551, 2013.

18. Sobin LH and Wittekind C: TNM Classification of Malignant Tumor. 6th edition. John Wiley and Sons, New Jersey, 2002.

19. Thöny B, Auerbach G and Blau N: Tetrahydrobiopterin biosynthesis, regeneration and functions. Biochem J 347: 1-16, 2000.

20. Werner ER, Blau N and Thöny B: Tetrahydrobiopterin: Biochemistry and pathophysiology. Biochem J 438: 397-414, 2011.

21. Chen L, Zeng X, Kleibeuker E, Buffa F, Barberis A, Leek RD, Roxanis I, Zhang W, Worth A, Beech JS, et al: Paracrine effect of GTP cyclohydrolase and angiopoietin-1 interaction in stromal fibroblasts on tumor Tie2 activation and breast cancer growth. Oncotarget 7: 9353-9367, 2016.

22. Trojanowicz B, Brodauf L, Sekulla C, Lorenz K, Finke R, Dralle $\mathrm{H}$ and Hoang-Vu C: The role of AUF1 in thyroid carcinoma progression. Endocr Relat Cancer 16: 857-871, 2009.
23. Zucconi BE and Wilson GM: Modulation of neoplastic gene regulatory pathways by the RNA-binding factor AUF1. Front Biosci (Landmark Ed) 16: 2307-2325, 2011.

24. Vázquez-Chantada M, Fernández-Ramos D, Embade N, Martínez-Lopez N, Varela-Rey M, Woodhoo A, Luka Z, Wagner C, Anglim PP, Finnell RH, et al: HuR/methyl-HuR and AUF1 regulate the MAT expressed during liver proliferation, differentiation, and carcinogenesis. Gastroenterology 138: 1943-1953, 2010

25. Antoniades C, Shirodaria C, Van Assche T, Cunnington C, Tegeder I, Lötsch J, Guzik TJ, Leeson P, Diesch J, Tousoulis D, et al: GCH1 haplotype determines vascular and plasma biopterin availability in coronary artery disease effects on vascular superoxide production and endothelial function. J Am Coll Cardiol 52: 158-165, 2008.

26. Douglas G, Hale AB, Crabtree MJ, Ryan BJ, Hansler A, Watschinger K, Gross SS, Lygate CA, Alp NJ and Channon KM: A requirement for Gch1 and tetrahydrobiopterin in embryonic development. Dev Biol 399: 129-138, 2015.

27. Ying L and Hofseth LJ: An emerging role for endothelial nitric oxide synthase in chronic inflammation and cancer. Cancer Res 67: 1407-1410, 2007.

28. Loibl S, von Minckwitz G, Weber S, Sinn HP, Schini-Kerth VB, Lobysheva I, Nepveu F, Wolf G, Strebhardt K and Kaufmann M: Expression of endothelial and inducible nitric oxide synthase in benign and malignant lesions of the breast and measurement of nitric oxide using electron paramagnetic resonance spectroscopy. Cancer 95: 1191-1198, 2002.

29. Thomsen LL, Miles DW, Happerfield L, Bobrow LG, Knowles RG and Moncada S: Nitric oxide synthase activity in human breast cancer. Br J Cancer 72: 41-44, 1995.

30. Beevi SS, Rasheed MH and Geetha A: Evidence of oxidative and nitrosative stress in patients with cervical squamous cell carcinoma. Clin Chim Acta 375: 119-123, 2007.

31. Masri FA, Comhair SA, Koeck T, Xu W, Janocha A, Ghosh S, Dweik RA, Golish J, Kinter M, Stuehr DJ, et al: Abnormalities in nitric oxide and its derivatives in lung cancer. Am J Respir Crit Care Med 172: 597-605, 2005.

32. Feng CW, Wang LD, Jiao LH, Liu B, Zheng S and Xie XJ: Expression of p53, inducible nitric oxide synthase and vascular endothelial growth factor in gastric precancerous and cancerous lesions: Correlation with clinical features. BMC Cancer 2: 8 , 2002.

33. Cooper RG and Magwere T: Nitric oxide-mediated pathogenesis during nicotine and alcohol consumption. Indian J Physiol Pharmacol 52: 11-18, 2008

34. Chen L, Zeng X, Wang J, Briggs SS, O'Neill E, Li J, Leek R, Kerr DJ, Harris AL and Cai S: Roles of tetrahydrobiopterin in promoting tumor angiogenesis. Am J Pathol 177: 2671-2680, 2010. 